\title{
Detection of retinal vascular bifurcations by rotation-, scale- and reflection-invariant COSFIRE filters
}

\author{
George Azzopardi and Nicolai Petkov \\ Johann Bernoulli Institute for Mathematics and Computer Science \\ University of Groningen, The Netherlands. \\ \{g.azzopardi, n.petkov\}@ rug.nl
}

\begin{abstract}
We propose trainable filters, which we call COSFIRE (Combination Of Shifted FIlter REsponses), and use to detect vascular bifurcations in retinal images. We configure a COSFIRE filter to be selective for a bifurcation that is specified by a user in a single-step training phase. The automatic configuration comprises the selection of channels of a bank of Gabor filters and the determination of certain blur and shift parameters. A COSFIRE filter response is computed as the geometric mean of the blurred and shifted responses of the selected Gabor filters. The proposed filters share similar properties with some shape-selective neurons in visual cortex. With only five filters we achieve a recall of $98.57 \%$ at a precision of $95.37 \%$ on the 40 binary retinal images (from DRIVE), containing more than 5000 bifurcations.
\end{abstract}

\section{Introduction}

Retinal fundus images provide a unique possibility to analyse the state of the vascular system of a person in a non-invasive way. The analysis of the geometrical structure of the vessel tree is important as deviations from the optimal principles [10] may indicate risks for some cardiovascular diseases, such as hypertension [15] and atherosclerosis [5]. The identification of vascular bifurcations is a crucial step in this analysis, which is typically performed by a tedious manual process [5].

The existing attempts to automate the detection of vascular bifurcations can be categorized into two classes: geometrical-feature based and model based approaches. The former involves extensive preprocessing, such as segmentation and skeletonization, followed by local pixel processing. These techniques are robust to the localization of bifurcations [3, 4, 6, 9]. Model based approaches are usually more adaptive and have lower computational complexity $[1,14]$. However, these approaches are known to suffer from insufficient generalization ability and consequently, they may fail to detect some relevant features.

We propose COSFIRE (Combination Of Shifted FIlter REsponses) filters for the detection of vascular bifurcations in retinal images. A COSFIRE filter is trainable as it is configured to be selective for a given prototype bifurcation. We demonstrate that a COSFIRE filter can achieve invariance to some geometrical transformations and is able to detect features that are similar to the prototype bifurcation.

The rest of the paper is organized as follows: in Section 2 we present the COSFIRE filter and in Section 3 we evaluate the proposed approach by applying COSFIRE filters on the DRIVE data set [13], which is a popular data set in the field of retinal image analysis. Finally, we provide a discussion and draw conclusions in Section 4.

\section{Proposed method}

Fig. 1a illustrates a typical bifurcation encircled in a binarized retinal image ${ }^{1}$. We use this feature, which is shown enlarged in Fig.1b, to automatically configure a COSFIRE filter that will respond to the same and similar bifurcations.

The three ellipses shown in Fig.1b illustrate the dominant orientations in the surroundings of the point of interest. We use 2D Gabor filters to detect these orientations. The central circle represents the overlapping supports of a group of such Gabor filters. The response of a COSFIRE filter is computed by combining the responses of the concerned Gabor filters by geometric mean.

We use an automatic configuration process to determine the preferred wavelengths and orientations of these Gabor filters and the locations at which we take their responses. Taking the Gabor responses at different locations around a point can be implemented by shifting the responses of these Gabor filters appropriately before evaluating them by a pixel-wise function that gives the COSFIRE filter output.

\footnotetext{
${ }^{1}$ The image is named 21_manual1.gif in the DRIVE data set [13].
} 


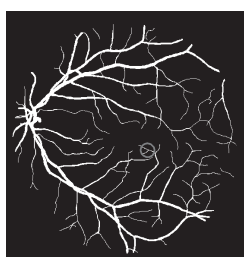

(a)

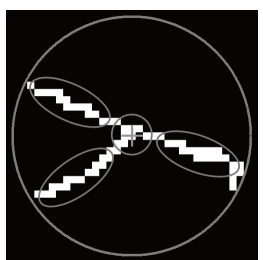

(b)

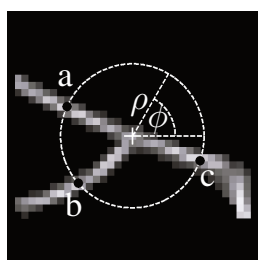

(c)

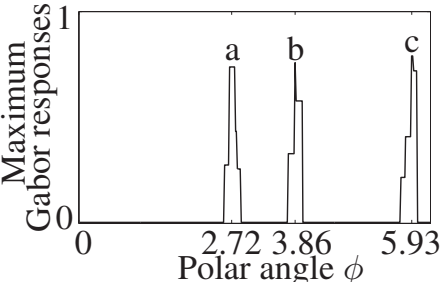

(d)

Figure 1. (a) The circle indicates a vascular bifurcation that is selected by a user. (b) The ellipses represent the dominant orientations around the specified point of interest, which is indicated by the ' + ' marker. (c) Superposition of the responses of a bank of Gabor filters. (d) Values of the Gabor responses along the bright circle of a given radius $\rho$ (here $\rho=10$ ) shown in (c). The labeled black dots in (c) mark the positions (relative to the center of the filter) of three local maxima points.

\subsection{Gabor filters: detecting orientations}

We denote by $\left|g_{\lambda, \theta}(x, y)\right|_{t_{1}}$ the half-wave rectified response of a symmetric Gabor filter of preferred wavelength $\lambda$ and orientation $\theta$ to a given input image. Such a filter has other parameters, which we set as proposed in [12].

We apply a bank of Gabor filters with five wavelengths $\left(\lambda \in\left\{4\left(2^{\frac{i}{2}}\right) \mid i=0 \ldots 4\right\}\right)$ and eight orientations $(\theta \in$ $\left.\left\{\frac{\pi i}{8} \mid i=0 \ldots 7\right\}\right)$ to retinal images of size $565 \times 584$ pixels, the blood vessels of which have a width that vary between 1 and 7 pixels. We threshold the Gabor responses at a fraction $t_{1}=0.2$ of the maximum response. Fig. 1c illustrates the superimposed thresholded responses of the bank of Gabor filters obtained for the bifurcation image shown in Fig. 1b.

\subsection{Configuration of a COSFIRE filter}

We consider the responses of the bank of Gabor filters along a circle ${ }^{2}$ of a given radius $\rho$ around the point of interest, Fig.1(c-d). In each position along that circle, we take the maximum of all responses across the possible values of $(\lambda, \theta)$. The positions that have values greater than the corresponding values of the neighbouring positions along an arc of angle $\pi / 8$ are selected as the points that characterize the dominant orientations. We determine the polar coordinates $(\rho, \phi)$ for each such a point with respect to the center of the filter. For such a location $(\rho, \phi)$ we then consider all combinations of $(\lambda, \theta)$ for which the corresponding responses $g_{\lambda, \theta}(x, y)$ are greater than a fraction $t_{2}=0.75$ of the maximum response. For each value $\theta$ that satisfies this condition, we consider a single value of $\lambda$, the one for which $g_{\lambda, \theta}(x, y)$ is the maximum of all responses across all values of $\lambda$. For each distinct pair of $(\lambda, \theta)$ and for location $(\rho, \phi)$ we obtain a tuple $(\lambda, \theta, \rho, \phi)$.

We denote by $S_{f}=\left\{\left(\lambda_{i}, \theta_{i}, \rho_{i}, \phi_{i}\right) \mid i=1 \ldots n_{f}\right\}$ the set of parameter value combinations, which fulfill the above

\footnotetext{
${ }^{2}$ For $\rho=0$ we only consider the point of interest.
}

conditions. The subscript $f$ stands for the vascular bifurcation around the selected point of interest. Every tuple in the set $S_{f}$ specifies the parameters of some contour part in $f$.

For the point of interest shown in Fig.1c and two values of the parameter $\rho(\{0,10\})$, the automatic configuration process results in four contour parts with parameter values specified by the tuples in the following set: $S_{f}=\left\{\left(\lambda_{1}=\right.\right.$ $\left.4, \theta_{1}=\frac{3 \pi}{8}, \rho_{1}=0, \phi_{1}=0\right),\left(\lambda_{2}=4, \theta_{2}=\frac{3 \pi}{8}, \rho_{2}=10, \phi_{2}=\right.$ $2.72),\left(\lambda_{3}=4, \theta_{3}=\frac{5 \pi}{8}, \rho_{3}=10, \phi_{3}=3.86\right),\left(\lambda_{4}=4, \theta_{4}=\right.$ $\left.\frac{3 \pi}{8}, \rho_{4}=10, \phi_{4}=5.93\right)$.

\subsection{Blurring and shifting Gabor responses}

We use the Gabor responses, the channels and relative locations of which are determined in the set $S_{f}$, to compute the output of the COSFIRE filter. We shift these responses appropriately so that they meet at the filter center before evaluating them by a pixel-wise multivariate function that gives the COSFIRE filter output.

Before these shift operations, we blur the Gabor responses to allow for some tolerance in the position of the respective contour parts. We define the blurring operation as the computation of maximum value of the weighted Gabor responses. For weighting we use a Gaussian function $G_{\sigma}(x, y)$ with a standard deviation $\sigma$ defined as: $\sigma=$ $0.67+0.1 \rho$. In practice, we implement this computation as $\max _{x^{\prime}, y^{\prime}}\left\{g_{\lambda, \theta}\left(x-x^{\prime}, y-y^{\prime}\right) G_{\sigma}\left(x^{\prime}, y^{\prime}\right)\right\}$ where $-3 \sigma \leq x^{\prime}, y^{\prime} \leq 3 \sigma$.

Next, we shift the blurred responses of each selected Gabor filter $\left(\lambda_{i}, \theta_{i}\right)$ by a vector $\left(\rho_{i}, \phi_{i}+\pi\right)$. We denote by $s_{\lambda_{i}, \theta_{i}, \rho_{i}, \phi_{i}}(x, y)$ the blurred and shifted response of the Gabor filter that is specified by the $i$-th tuple in the set $S_{f}$. In practice, the computation of one blurred and shifted response (for the same values of $\lambda, \theta$ and $\rho$ ), for instance with $s_{\lambda, \theta, \rho, \phi=0}(x, y)$, is sufficient: the result of $s_{\lambda, \theta, \rho, \phi}(x, y)$ for any value of $\phi$ can be obtained from the result of the output of $s_{\lambda, \theta, \rho, \phi=0}(x, y)$ by appropriate shifting. 


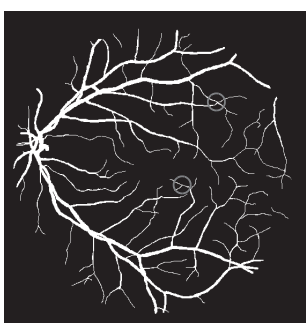

(a) $\mathbf{T P}=2$

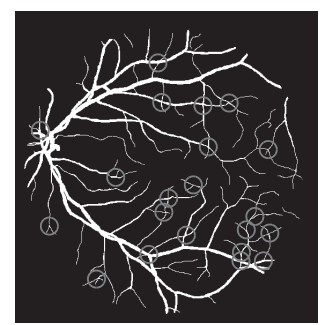

(b) $\mathbf{T P}=21$

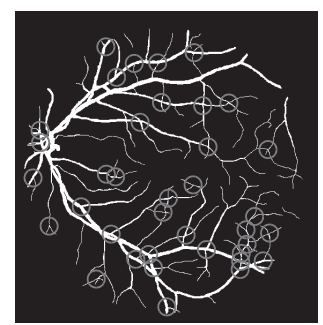

(c) $\mathbf{T P}=37$

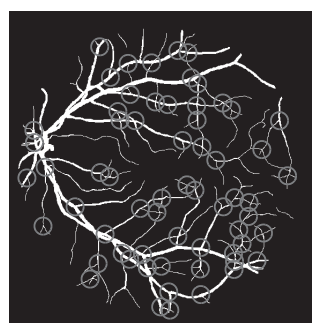

(d) $\mathrm{TP}=63$

Figure 2. Applying filter $S_{f_{1}}$ in different modes: (a) non-invariant, (b) rotation-invariant, (c) rotationand scale-invariant, and (d) rotation-, scale- and reflection-invariant. The number of detected true positive (TP) features increases as the filter achieves invariance to such geometric transformations.

\subsection{COSFIRE filter output}

We denote by $r_{S_{f}}(x, y)$ the response of a COSFIRE filter that we compute as the geometric mean of all the blurred and shifted Gabor responses $s_{\lambda_{i}, \theta_{i}, \rho_{i}, \phi_{i}}(x, y)$ for the set $S_{f}$.

$$
r_{S_{f}}(x, y)=\left|\left(\prod_{i=1}^{\left|S_{f}\right|} s_{\lambda_{i}, \theta_{i}, \rho_{i}, \phi_{i}}(x, y)\right)^{\frac{1}{\left|S_{f}\right|}}\right|_{t_{3}}
$$

where $|\cdot|_{t_{3}}$ stands for thresholding the response at a fraction $t_{3}$ of its maximum.

Rotation and scale invariance are achieved as follows. Using the set $S_{f}$, we first form a new set $\Re_{\psi, v}\left(S_{f}\right)=$ $\left\{v \lambda_{i}, \theta_{i}+\psi, v \rho_{i}, \phi_{i}+\psi|\forall i \in 1 \ldots| S_{f} \mid\right\}$, and then we define the rotation- and scale-invariant response as $\widehat{r}_{S_{f}}(x, y)=$ $\max _{\psi, v}\left(r_{\Re_{\psi, v}\left(S_{f}\right)}(x, y)\right)$. We achieve reflection invariance by forming another set: $S_{f}^{\prime}=\left\{\lambda_{i}, \pi-\theta_{i}, \rho_{i}, \pi-\phi_{i}\right) \mid \forall i \in$ $\left.1 \ldots\left|S_{f}\right|\right\}$. The new filter $S_{f}^{\prime}$ is selective for a mirrored reflection, about the $y$-axis, of the prototype pattern $f$.

Finally, we define the rotation-, scale- and reflectioninvariant response of a COSFIRE filter $S_{f}$ as the maximum value of $\widehat{r}_{\Re_{\psi, v}\left(S_{f}\right)}(x, y)$ and $\widehat{r}_{\Re_{\psi, v}\left(S_{f}^{\prime}\right)}(x, y)$.

\section{Experimental results}

We use the prototype bifurcation in Fig.1 to configure a filter denoted by $S_{f_{1}}$ with three values of $\rho(\rho \in\{0,4,10\})$. Fig. 2 shows the results that are obtained by the application of filter $S_{f_{1}}$ in different modes. For this filter we use a threshold $t_{3}=0.37$ as it produces the largest number of correctly detected bifurcations and no falsely detected features. The encircled regions are centered on the local maxima of the filter response and if two such regions overlap by $75 \%$, only the one with the stronger response is shown.

When no invariance is used (Fig. 2a), the filter $S_{f_{1}}$ detects two vascular bifurcations one of which is the proto-

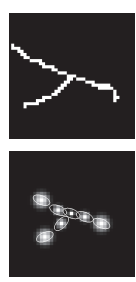

$\mathbf{S}_{\mathbf{f}_{1}}$
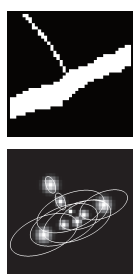

$\mathrm{S}_{\mathrm{f}_{2}}$
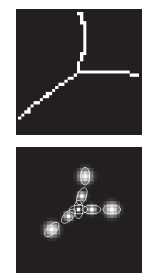

$\mathrm{S}_{\mathbf{f}_{3}}$
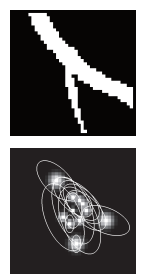

$\mathrm{S}_{\mathrm{f}_{4}}$

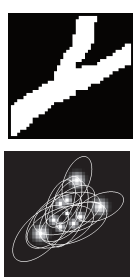

$\mathrm{S}_{\mathrm{f}_{5}}$
Figure 3. (Top row) Selected vascular bifurcations and (bottom row) the structures of the corresponding COSFIRE filters.

type pattern that was used to configure $S_{f_{1}}$. If the filter is applied in a rotation-invariant mode $\left(\psi \in\left\{\frac{\pi i}{8} \mid i=0 \ldots 7\right\}\right)$ it detects 21 features. With the addition of scale-invariance $\left(v \in\left\{2^{-\frac{1}{2}}, 1,2^{\frac{1}{2}}\right\}\right)$ the filter detects 37 features, and finally with the inclusion of reflection-invariance, it detects 63 bifurcations. This example illustrates the strong generalization ability of this approach because $58.88 \%$ (63 out of 107) of interest are detected by a single filter.

As to the remaining features that are not detected by this filter, we proceed as follows: we use one of these features that we denote by $f_{2}$ to train a second COSFIRE filter $S_{f_{2}}$, Fig. 3. With the new filter we detect 40 features of interest of which 22 coincide with features detected by filter $S_{f_{1}}$ and 18 are newly detected features $\left(t_{3}\left(S_{f_{2}}\right)=0.44\right)$. Merging the responses of both filters results in the detection of 81 features. By configuring another three COSFIRE filters, $S_{f_{3}}, S_{f_{4}}$ and $S_{f_{5}}$ (Fig. 3), and use them $\left(t_{3}\left(S_{f_{3}}\right)=\right.$ $\left.0.14, t_{3}\left(S_{f_{4}}\right)=0.27, t_{3}\left(S_{f_{5}}\right)=0.37\right)$ together with the other two filters we achieve $100 \%$ recall and $100 \%$ preci$\operatorname{sion}^{3}$ for the concerned image.

We use a threshold value $t_{3}\left(S_{f_{i}}\right)$ for each COSFIRE filter $S_{f_{i}}$ by automatically setting it to the smallest fraction for which the precision is still $100 \%$ for the training image.

\footnotetext{
${ }^{3}$ Recall is the percentage of true features that are successfully detected. Precision is the percentage of correct features from all detected patterns.
} 
We apply these five COSFIRE filters on all the 40 binary retinal images ${ }^{4}$ of the DRIVE data set and evaluate the obtained results with the publicly available ground truth data ${ }^{5}$. We achieve a total recall of $99.04 \%$ and a total precision of $91.63 \%$. We then evaluate the filters by repeatingly changing their corresponding learned thresholds $t_{3}\left(S_{f_{i}}\right)$ with a given offset value. The maximum of the harmonic mean ${ }^{6}$ is reached for a recall of $98.57 \%$ and a precision of $95.37 \%$.

\section{Discussion and conclusion}

With only five COSFIRE filters we achieve $98.57 \%$ recall and $95.37 \%$ precision on the DRIVE data set of 40 binary retinal images, which contains more than 5000 bifurcations. These results outperform the ones reported in [2] that were achieved with 25 trainable filters $(98.52 \%$ recall and $95.19 \%$ precision), the responses of which were thresholded at the same value. In [3] a recall of $95.82 \%$ is reported on a small data set of five retinal images.

The proposed COSFIRE filters are trainable, in that the structure of such a filter is automatically determined by a prototype pattern that is specified by a user. We use symmetric Gabor filters for detecting blood vessels. Gabor filters are, however, not intrinsic to the proposed method, and other orientation-selective filters can also be used.

A COSFIRE filter share similar properties with some neurons in area V4 of visual cortex which exhibit selectivity for parts of (curved) contours [11]. We compute the filter response by the geometric mean which is inspired by psychophysical evidence that human visual processing of shape is likely performed by multiplication [7].

A COSFIRE filter produces a response only when all constituent parts of a pattern of interest are present. This is in contrast to other feature detection approaches, such as SIFT [8], in which two patterns are considered similar if the Euclidean distance between their respective local descriptors satisfies certain criteria. Euclidean-distance based approaches, however, suffer from insufficient selectivity regarding the shape properties of features.

The implementation of a COSFIRE filter is simple: the filter output is computed as the geometric mean of blurred and shifted Gabor responses. COSFIRE filters are versatile as they can be configured with any local contour patterns.

In principle, all vascular bifurcations can be detected if a sufficient number of filters are configured and used. The performance that we obtain is sufficient to the needs of the medical application at hand. We conclude that the proposed rotation-, scale- and reflection-invariant COSFIRE filters are an effective means to automatically detect vascular bifurcations in retinal fundus images.

\footnotetext{
${ }^{4}$ Named in DRIVE 01_manual1.gif, ..., 40_manual1.gif.

${ }^{5}$ Ground truth data: www.cs.rug.nl/ imaging/databases/retina_database

${ }^{6}$ Harmonic mean of precision $P$ and recall $R$ is defined as: $\frac{2 P R}{P+R}$.
}

\section{References}

[1] C. Ali, S. Hong, J. Turner, H. Tanenbaum, and B. Roysam. Rapid automated tracing and feature extraction from retinal fundus images using direct exploratory algorithms. IEEE Transactions on Information Technology in Biomedicine, 3:125-138, 1999.

[2] G. Azzopardi and N. Petkov. Detection of Retinal Vascular Bifurcations by Trainable V4-Like Filters. In Computer Analysis of Images and Patterns. Proceedings 14th International Conference, CAIP 2011, number pt.1, pages 451-9.

[3] A. Bhuiyan, B. Nath, J. Chua, and K. Ramamohanarao. Automatic detection of vascular bifurcations and crossovers from color retinal fundus images. Third International IEEE Conference on Signal-Image Technologies and InternetBased System (SITIS), pages 711-718, 2007.

[4] T. Chanwimaluang and F. Guoliang. An efficient blood vessel detection algorithm for retinal images using local entropy thresholding. Proceedings of the 2003 IEEE International Symposium on Circuits and Systems (Cat. No.03CH37430), 5.

[5] N. Chapman, G. Dell'omo, M. Sartini, N. Witt, A. Hughes, S. Thom, and R. Pedrinelli. Peripheral vascular disease is associated with abnormal arteriolar diameter relationships at bifurcations in the human retina. Clinical Science, 103.

[6] J. Eunhwa and H. Kyungho. Automatic retinal vasculature structure tracing and vascular landmark extraction from human eye image. International Conference on Hybrid Information Technology, 7, 2006.

[7] E. Gheorghiu and F. Kingdom. Multiplication in curvature processing. Journal of Vision, 9, 2009.

[8] D. Lowe. Distinctive image features from scale-invariant keypoints. International Journal of Computer Vision, 60:91-110, 2004.

[9] M. Martinez-Perez, A. Hughes, A. Stanton, S. Thom, N. Chapman, A. Bharath, and K. Parker. Retinal vascular tree morphology: A semi-automatic quantification. IEEE Transactions on Biomedical Engineering, 49:912917, 2002.

[10] C. D. Murray. The physiological principle of minimum work applied to the angle of branching of arteries. 9:835-841, 1926.

[11] A. Pasupathy and C. Connor. Responses to contour features in macaque area v4. Journal of Neurophysiology, 82:2490 2502, 1999.

[12] N. Petkov. Biologically motivated computationally intensive approaches to image pattern-recognition. Future Generation Computer Systems, 11:451-465, 1995.

[13] J. Staal, M. Abramoff, M. Niemeijer, M. Viergever, and B. van Ginneken. Ridge-based vessel segmentation in color images of the retina. IEEE Transactions on Medical Imaging, 23:501-509, 2004.

[14] C. Tsai, C. Stewart, H. Tanenbaum, and B. Roysam. Modelbased method for improving the accuracy and repeatability of estimating vascular bifurcations and crossovers from retinal fundus images. IEEE Transactions on Information Technology in Biomedicine, 8:122-130, 2004.

[15] M. Tso and L. Jampol. Path-physiology of hypertensive retinopathy. Opthalmology, 89. 